\title{
Realtime photoacoustic microscopy of murine cardiovascular and respiratory dynamics in vivo
}

Roger J. Zemp, Liang Song, Rachel Bitton, K. Kirk Shung, Lihong V. Wang

Roger J. Zemp, Liang Song, Rachel Bitton, K. Kirk Shung, Lihong V. Wang, "Realtime photoacoustic microscopy of murine cardiovascular and respiratory dynamics in vivo," Proc. SPIE 6856, Photons Plus Ultrasound: Imaging and Sensing 2008: The Ninth Conference on Biomedical Thermoacoustics, Optoacoustics, and Acousto-optics, 68560G (29 February 2008); doi: 10.1117/12.763127

SPIE. Event: SPIE BiOS, 2008, San Jose, California, United States 


\title{
Realtime Photoacoustic Microscopy of Murine Cardiovascular and Respiratory Dynamics In Vivo
}

\author{
Roger J. Zemp ${ }^{\mathrm{a}, \mathrm{c}, 1}$, Liang Song ${ }^{\mathrm{a}}$, Rachel Bitton ${ }^{\mathrm{b}}$, K. Kirk Shung ${ }^{\mathrm{a}}$, Lihong V. Wang ${ }^{\mathrm{a}, 2}$ \\ ${ }^{a}$ Optical Imaging Laboratory, Department of Biomedical Engineering, Washington University in St. \\ Louis, St. Louis, Missouri 63130; \\ ${ }^{b}$ University of Southern California, Department of Biomedical Engineering, Los Angeles, California \\ 90089 \\ ${ }^{\mathrm{c} C u r r e n t}$ address: Department of Electrical and Computer Engineering, University of Alberta, \\ Edmonton, Alberta, Canada, T6G 2V4
}

\begin{abstract}
While photoacoustic imaging has emerged as a promising modality in recent years, a key drawback of practical and widespread use of the technique has been slow imaging rates. We present a 30-MHz array-based photoacoustic imaging system that can acquire and display photoacoustic images in realtime. Realtime display is very helpful and provides the system operator the ability to better navigate and position the probe for selecting a desired anatomical field of view. The system is capable of imaging at 50 frames per second to depths of a few $\mathrm{mm}$ in tissue. We used this system to successfully image the beating hearts of young athymic nude mice in vivo. Also of interest was the ability to visualize microvascular changes during respiration.
\end{abstract}

Keywords: photoacoustic imaging, high-frequency ultrasound, Q-switched lasers, biophotonics, cardiovascular research

\section{INTRODUCTION}

Few imaging techniques are able to visualize cardiovascular temporal dynamics in mice with high spatial and temporal resolution. The ability to visualize and quantify such dynamics, however, is of utmost importance in cardiovascular research. Because mice possess $99 \%$ genetic homology with humans, they are serving as important models of disease. Transgenic and knockout models of cardiovascular disease are enabling researchers to study genotype-phenotype relationships. Additionally, pharmaceutical development for cardiovascular diseases often use small animal models for testing. Unfortunately, cardiovascular small animal research often requires sacrificing animals at various time-points for ex vivo analysis. Inter-subject variability, however, complicates statistical analysis necessary to demonstrate drug efficacy or to validate the effects of some other controlled perturbation.

Imaging techniques potentially enable longitudinal studies permitting non-invasive measurements in one animal over time, and may reduce the number of animals in or accelerate the time for the research process. Small animal imaging in cardiovascular research is particularly challenging because of rapid heart-rates requiring high imaging frame-rates.

High-frequency ultrasound has recently emerged as a powerful tool for realtime high-resolution scanning [1], and has greatly impacted cardiovascular research. High-frequency B-mode imaging offers only structural information, while functional information about blood flow is available with Doppler techniques. Still, most high-frequency ultrasound systems for small animals use a mechanically-scanned single element transducer, due to challenges in fabricating highfrequency arrays. While rapid scanning mechanisms allow 30 -frames per second imaging rates, high frame rates typically mean less sensitivity to slow blood flows, a tradeoff partially remedied by inter-frame clutter filtering [2], but inevitably still requiring Doppler frame-rate sacrifices, presently $\sim 15$ fps. When small animal heart rates are 360 beats per minute (or 6 beats per second), this frame-rate is inadequate, and retrospective reconstruction of cardiac-gated

\footnotetext{
${ }^{1}$ Email: zemp@ece.ualberta.ca

${ }^{2}$ Corresponding author: $\underline{\text { hwwang}} @$,biomed.wustl.edu.
}

Photons Plus Ultrasound: Imaging and Sensing 2008: The Ninth Conference on Biomedical Thermoacoustics, Optoacoustics, and Acousto-optics, edited by Alexander A. Oraevsky, Lihong V. Wang, Proc. of SPIE Vol. 6856, 68560G, (2008) · 1605-7422/08/\$18 - doi: 10.1117/12.763127 
acquisitions may be required. Beyond frame-rate limitations, the cardiovascular research community could surely benefit from an imaging modality providing a new functional contrast mechansism.

Biophotonic imaging promises the visualization of internal structures in biological tissues with optical contrast, revealing molecular and functional information. Unfortunately the highly scattering nature of light in tissues greatly complicates efforts to produce high-resolution images for imaging depths below a transport mean-free path. While diffuse-optical tomographic methods permit and rely on multiply-scattered light, their reconstruction methods are ill-posed, transportregime reconstruction is challenging [3,4], and fine spatial resolution is practically difficult to obtain due to factors such as signal-to-noise and regularization.

Photoacoustic imaging is an emerging technology that partially circumvents the problem that scattering poses to imaging resolution. Short laser pulses (typically on the order of nanoseconds) are fired into tissue and because the pulse-lengths are brief, light absorption in subcutaneous pigments is thermally confined, producing rapid but minimal heating, and causing temperature-induced expansion. The thermo-elastic excitation from chromophores launches ultrasonic pressure waves which are detected by an ultrasound transducer in the imaging system. The received photoacoustic signals are subsequently reconstructed to form images of subcutaneous optical absorption. Spatial resolution in this case is determined primarily by ultrasonic focusing and receiver frequency response, and multiply-scattered light is welltolerated.

Analogous to computed tomography, circular-scanning photoacoustic tomography (PAT) has been used for non-invasive visualization of small animal brains [5, 6]. Maslov et al [7] and Zhang et al [8] have demonstrated images of subcutaneous microvasculature using dark-field confocal photoacoustic microscopy (PAM), a raster-scanning-based reflection geometry imaging system. Leveraging known oxy-hemoglobin and deoxy-hemoglobin absorption spectra, photoacoustic methods possess the ability to image blood oxygen saturation as well as relative concentration of total hemoglobin [8-10]. Emerging photoacoustic methods are enabling molecular imaging in small animals, including imaging of gene expression [11] and cell receptors[12]. Unfortunately, long scan times are typically required in both scanning PAT and PAM.

Our goal was to construct a realtime high-frequency photoacoustic imaging system. In principle, using an ultrasound array transducer, a photoacoustic image can be formed from each laser shot by reconstructing photoacoustic signals received in parallel through array elements. The system described in this article approaches this goal but rather uses a small number of laser shots at a high-repetition rate to acquire signals in multiplexed mode. Even with the present multiplexing scheme, our system offers realtime performance.

Although Niederhauser et al [13] demonstrated photoacoustic imaging with a linear ultrasound array, their system lacked realtime imaging capabilities. Moreover, they used only 16 receive elements to form an image using a diagnostic frequency ultrasound array. Others $[14,15]$ have also demonstrated the use of arrays for photoacoustic imaging, however for low diagnostic frequencies. Optical detection of ultrasound using Fabry-Perot etalons has led to emerging 2-D arrays, recently producing 3-D microvascular images in animals and human subjects [16]. While this technique offers considerable promise, the signal-to-noise ratio strongly depends on the laser power of a laser probe beam, and presently this often means the necessity of scanning the probe beam, necessitating many pulsed laser excitations, and sacrificing realtime performance. A high-frequency array system for photoacoustic microscopy was previously reported by our group [17], but realtime acquisition and display was lacking. To our knowledge, this is the first published report of a realtime high-frequency photoacoustic imaging system.

We demonstrate the realtime imaging capabilities of our system by imaging the beating hearts of young athymic nude mice. We also demonstrate the ability to visualize changes in microvascular volume due to respiration.

\section{METHODS}

Our system consisted of a 30-MHz ultrasound array transducer, a KHz-repetition-rate Q-switched laser, custom receive electronics, an 8-channel digitizer, and a multi-core processor computer, as shown in Fig. 1. 


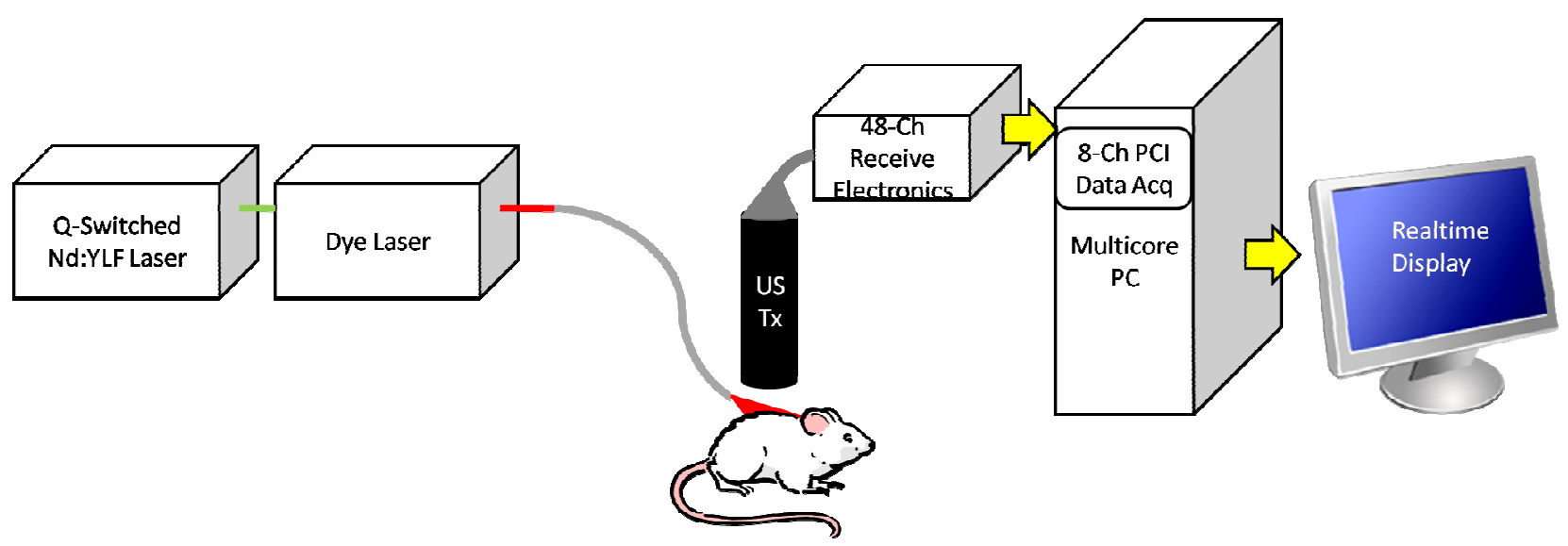

Figure 1. Diagram of our realtime photoacoustic microscopy system.

\subsection{High-Frequency Ultrasound Array Transducer}

We used a unique $30-\mathrm{MHz}$ linear array transducer to receive photoacoustic signals. The array was fabricated from a piezo-composite by the NIH Transducer Resource Center at the University of Southern California [18]. Our array possessed 48 elements with $100-\mu \mathrm{m}$ pitch $(2 \lambda), 19.1 \mathrm{~dB}$ compensated pulse-echo insertion loss, and element cross-talk below $40 \mathrm{~dB}$. An acoustic lens provided a fixed $8.2 \mathrm{~mm}$ elevation focus with elements possessing 2-mm height.

\subsection{Laser and Light Delivery}

We used a Nd:YLF diode-pumped Q-switched laser (INNOSLAB, Edgewave GmbH, Germany) to produce 6-ns optical pulses of $\sim 14 \mathrm{~mJ}$ at a wavelength of $524 \mathrm{~nm}$. This laser permitted external triggering at rates up to $1 \mathrm{KHz}$, without pulse energy loss. For our imaging system, this feature is a significant advantage over flashlamp-pumped Qswitched lasers. This laser was used to pump a dye laser (Cobra, Sirah Laser - und Plasmatechnik GmbH, Germany). The 2-3 mJ of dye laser output were coupled into a $600-\mu \mathrm{m}$ optical fiber and light from the other end of the fiber was delivered obliquely past the ultrasound transducer array forming an oblong pattern on the skin along the array axis. The estimated fluence was $5-10 \mathrm{~mJ} / \mathrm{cm}^{2}$ per pulse. For our imaging experiments we used $578-\mathrm{nm}$ and $571-\mathrm{nm}$ wavelengths corresponding to the peak dye laser power and an isosbestic point, respectively.

\subsection{Data Acquisition and Control Electronics}

A custom receiver-board, described by Bitton et al [19] was used to pre-amplify signals on each of the 48 channels, down-multiplex 3:1, bandpass filter, then amplify using a $40 \mathrm{~dB}$ variable gain amplifier (AD8332, Analog Devices). RF transformers are used to convert the differential output of the VGAs to a single line and a final $23 \mathrm{~dB}$ fixed gain stage offers a total variable gain between 33 and $73 \mathrm{~dB}$. All these steps were implemented on an RF printed circuit board having 48 SMA inputs and 16 SMA outputs. The remaining 16 channels were down-multiplexed to 8 channels using modified MAX4141 multiplexer evaluation boards (Maxim, Inc).

The post-multiplexed 8 lines were digitized at 125 MSPS using a 14-bit 8-channel PCI data acquisition card (Octopus CompuScope 8389, GaGe Applied Systems, USA). The card had 128 MB of on-board memory, and provided data transfer to the PC RAM at better than 200 MBPS. Benchmark software written in C showed the system capable of sustained data acquisition and transfer to PC RAM for $1 \mathrm{KS}$ records at roughly $1 \mathrm{KHz}$. For each B-frame to be acquired, 
the Gage card was programmed to record a sequence of 6 acquisitions, enabling multiplexed access to 48 array elements with 8 receive channels. A 1-KHz repetition rate for the 6 acquisitions per B-frame, with the precision of the onboard $125 \mathrm{MS} / \mathrm{s}$ clock. The inter-frame delay, was software controlled, and hence variable due to lack of interrupt control in the Windows operating system. We hypothesized that since a B-frame was acquired in only $6 \mathrm{~ms}$, beamforming degradation due to motion over multiplexed data acquisition should be minimal.

The multiplexer control circuit consisted of a simple 4-bit counter, counting from binary 0 to 5 . The counter was implemented using CMOS pre-settable counter integrated circuits (CD74HCT163E, Texas Instruments).

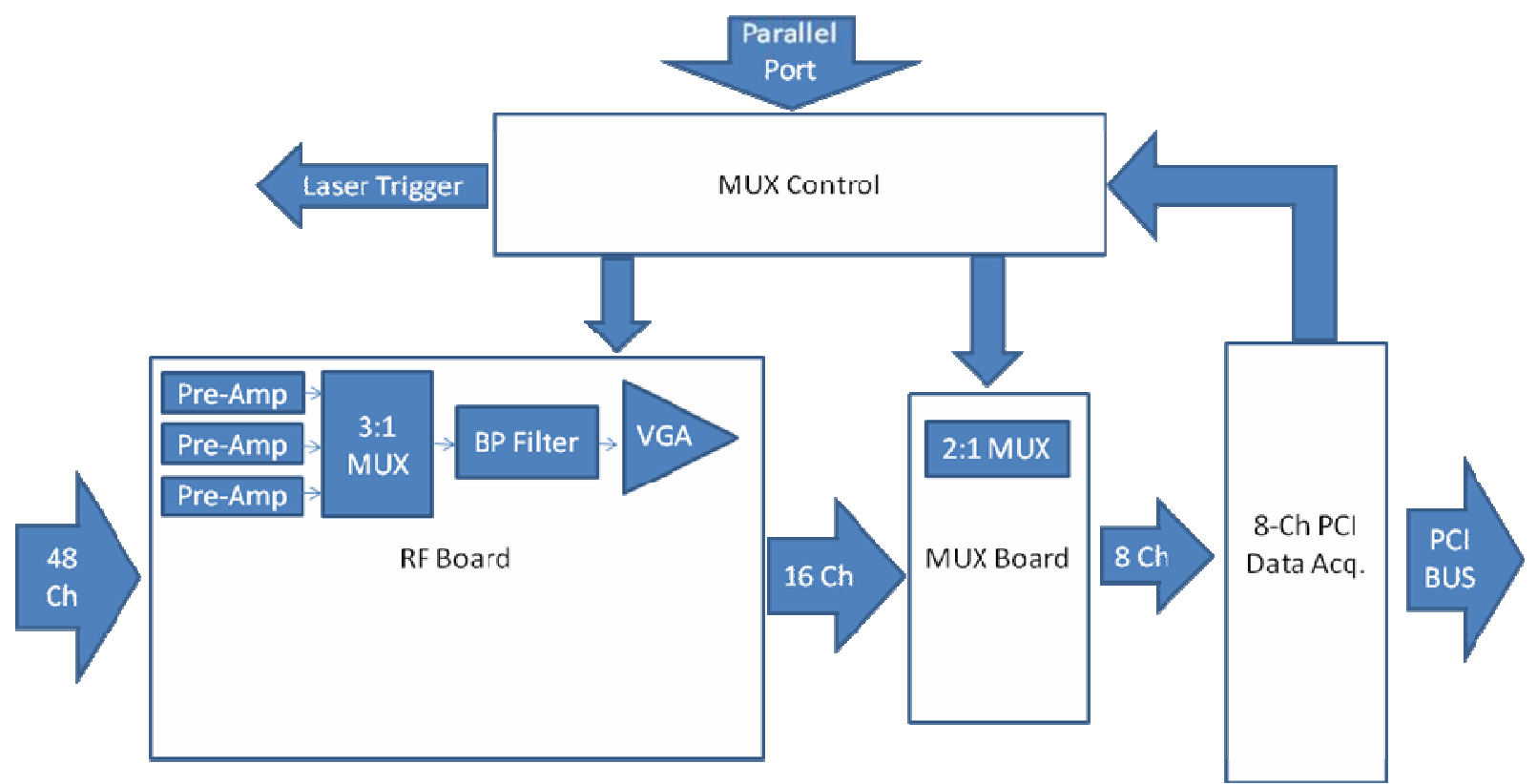

Fig. 2 Architecture of the receive and control electronics of our realtime photoacoustic imaging system. The 48 receive channels are boosted by pre-amplifiers, down-multiplexed (MUX), bandpass (BP) filtered, and amplified with a variable gain amplifier stage (VGA). The resulting 16 channels are down-multiplexed to 8 channels for digitization. Control electronics toggle through multiplexer states, and pass trigger signals to the laser.

\subsection{Software for realtime beamforming and display}

Data streamed via the data acquisition card to the PC RAM was beamformed in realtime using a dual-socket quad-core Xeon processor. Parallel programming methods, leveraging OpenMP pragmas in C++ Using Microsoft Visual Studio 2005 Professional Edition. Beamforming routines were compiled to Dynamic Link Libraries (.dll's) and called in the C\# environment during runtime. We used the $\mathrm{C} \#$ environment to take advantage of a programming environment called XNA game studio, to enable realtime scan conversion and display. Rather than implement scan conversion using CPU resources, we offloaded this task to the graphical processing unit of the video card. Scan conversion was accomplished by forming a pre-defined curvilinear $32 \times 32$ mesh, and warping the pre-scan-converted beamformed and envelopedetected data over the mesh.

\section{RESULTS}

\subsection{Resolution Studies}

To assess the spatial resolution of our system, we constructed a phantom consisting of crossed 6- $\mu \mathrm{m}$ carbon fibers. Sequential B-scans were acquired to form a 3-D dataset, and the maximum amplitude along each depth-line was projected to form a C-scan image, as shown in Fig. 3(a). Fig. 3(c) shows a the maximum cross-range amplitude of a B- 
scan at a position close to the fiber intersection. This shows clearly resolvable point-spread functions at $80-\mu \mathrm{m}$ separation distance.
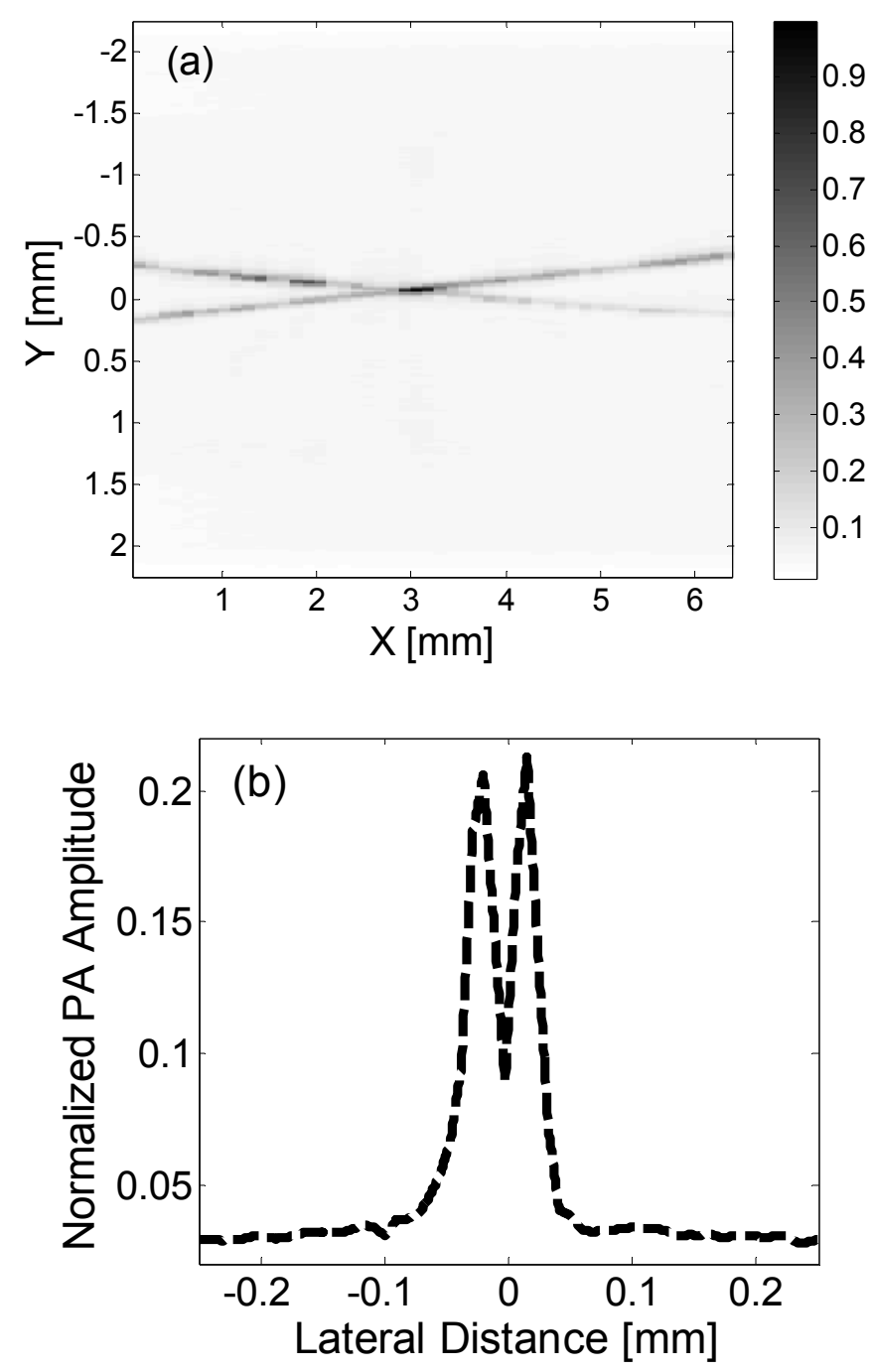

Fig. 3 Maximum amplitude C-scan image of two crossed 6-mm carbon fibers in water. (b) the crossrange maximum amplitude of a B-scan near the crossing-point of the two fibers. The two fibers are clearly separable at $80 \mathrm{~mm}$ separation distance.

\subsection{Animal Studies}

Young athymic nude mice $(\sim 10 \mathrm{~g})$ were anesthetized and sustained under anesthesia using a gas anesthesia machine. They were positioned in supine position with their fore-and hind-paws secured to a lab-jack with an adhesive. Acoustic coupling gel or a thin layer of water was applied to their thoracic region, and then the mice were raised up until they contacted a water-filled clear plastic membrane sagging below a water tank with a central rectangular aperture. The array and optical fiber were partially submerged in the water with a custom holder attached via optical posts to a 3-axis translation stage used to precisely position the probe. 


\subsection{Visualizing Cardiovascular Dynamics}

The array was positioned until pulsating structures of the heart could be visualized on the realtime display. Structures were visible down to $\sim 3 \mathrm{~mm}$ below perceivable dermal microvasculature signatures. Here we show one representative frame from a movie sequence.

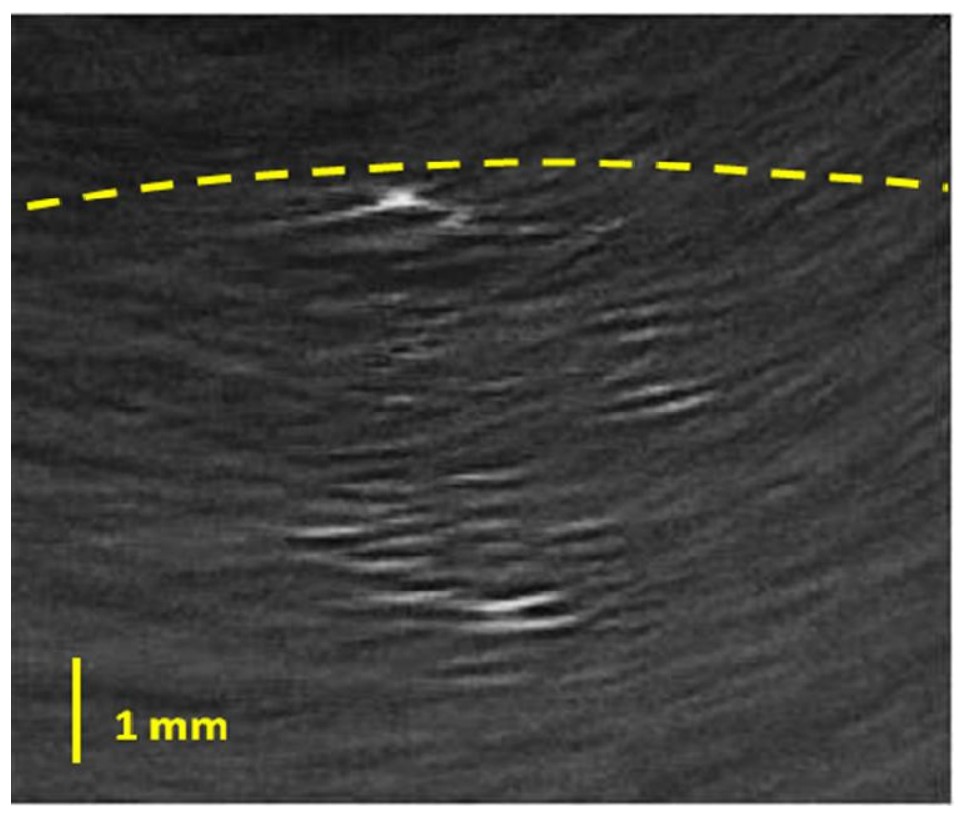

Figure 4. Photoacoustic B-scan of the upper thoracic region of a young athymic nude mouse.

\section{DISCUSSION}

The system presented here represents the first realtime high-frequency photoacoustic imaging system to our knowledge, and we have seen a glimpse of its capabilities. There is considerable room for forward progress and we now offer a discussion of such avenues.

Thus far, we have performed only single wavelength imaging. Future work may incorporate multiple-wavelength illumination for spectral estimation of blood oxygen saturation and other physiological parameters of interest. A fasttuning laser source would be highly desirable for this application.

For $<10 \mathrm{~s}$ dwell times, the maximum permissible exposure is limited by $1100 t_{e}^{1 / 4} \mathrm{in} \mathrm{mJ} / \mathrm{cm}^{2}$, where $t_{e}$ denotes the exposure duration in seconds. For our case, since we send a rapid train of 6 pulses followed by an inter-frame delay which is long enough for heat to adequately dissipate (as note above for $50 \mathrm{fps}$ rates), we take the exposure duration as 6 $\mathrm{ms}$. This corresponds to a total of $54.4 \mathrm{~mJ} / \mathrm{cm}^{2}$ delivered over the 6 pulses, translating into $\sim 9 \mathrm{~mJ} / \mathrm{cm}^{2}$ per pulse, which is roughly our present fluence.

\section{SUMMARY AND CONCLUSIONS}

We have demonstrated the first realtime photoacoustic imaging system to our knowledge. Using the system we have demonstrated non-invasive visualization of the beating hearts of young athymic nude mice with high spatial resolution. Very few imaging systems can do this. While emerging high-frequency ultrasound systems show this capability, functional imaging at these rates is challenging. Mechanically swept single element transducers can operate at 30 frames 
per second, but for Doppler imaging, there is a tradeoff between flow sensitivity and frame-rate. Moreover, Doppler ultrasound is not sensitive to blood oxygenation or other optical-absorption-dependent contrast.

\section{ACKNOWLEDGEMENTS}

We gratefully acknowledge helpful discussions with Dr. William D. Richard, who suggested the use of the dual-socket quad-core computers, and Dr. Robert E Morley Jr. and Mr. Ed Richter for allowing us to test the feasibility of our realtime beamforming algorithm on their dual-socket quad core workstations. We thank Dr. George Stoica for assistance with animal protocols. This work was funded in part by grants R01 EB000712 and R01 NS46214 from National Institutes of Health.

\section{REFERENCES}

[1] F.S. Foster, M.Y. Zhang, Y.Q. Zhou, G. Liu, J. Mehi, E. Cherin, K.A. Harasiewicz, B.G. Starkoski, L. Zan, D.A. Knapik, and S.L. Adamson, "A new ultrasound instrument for in vivo microimaging of mice," Ultrasound in Medicine and Biology, 28(9), 1165-1172 (2002).

[2] A. Needles, D.E. Goertz, A.M. Cheung, and F.S. Foster, "Interframe clutter filtering for high frequency flow imaging," Ultrasound in Medicine and Biology, 33(4), 591-600 (2007).

[3] A. Dunn and D. Boas, "Transport-based image reconstruction in turbid media with small source-detector separations," Optics Letters, 25(24), 1777-1779 (2000).

[4] E.M.C. Hillman, D.A. Boas, A.M. Dale, and A.K. Dunn, "Laminar optical tomography: demonstration of millimeter-scale depth-resolved imaging in turbid media," Optics Letters, 29(14), 1650-1652 (2004).

[5] G. Ku, X.D. Wang, X.Y. Xie, G. Stoica, and L.H.V. Wang, "Imaging of tumor angiogenesis in rat brains in vivo by photoacoustic tomography," Applied Optics, 44 (5), 770-775 (2005).

[6] X.D. Wang, Y.J. Pang, G. Ku, X.Y. Xie, G. Stoica, and L.H.V. Wang, "Noninvasive laser-induced photoacoustic tomography for structural and functional in vivo imaging of the brain," Nature Biotechnology, 21(7), 803-806 (2003).

[7] K. Maslov, G. Stoica, and L.V.H. Wang, "In vivo dark-field reflection-mode photoacoustic microscopy," Optics Letters, 30 (6), 625-627 (2005).

[8] H.F. Zhang, K. Maslov, G. Stoica, and L.H.V. Wang, "Functional photoacoustic microscopy for high-resolution and noninvasive in vivo imaging," Nature Biotechnology, 24(7), 848-851 (2006).

[9] M. Sivaramakrishnan, K. Maslov, H.F. Zhang, G. Stoica, and L.V. Wang, "Limitations of quantitative photoacoustic measurements of blood oxygenation in small vessels," Physics in Medicine and Biology, 52(5), 1349-1361 (2007).

[10] X.D. Wang, X.Y. Xie, G.N. Ku, and L.H.V. Wang, "Noninvasive imaging of hemoglobin concentration and oxygenation in the rat brain using high-resolution photoacoustic tomography," Journal of Biomedical Optics, 11(2), - (2006).

[11] L. Li, R.J. Zemp, G. Lungu, G. Stoica, and L.H.V. Wang, "Photoacoustic imaging of lacZ gene expression in vivo," Journal of Biomedical Optics, 12(2), -(2007).

[12] M. Li, J. Oh, X. X., G. Ku, W. Wang, C. Li, G. Lungu, G. Stoica, and L.V. Wang, "Simultaneous molecular and hypoxia imaging of brain tumors in vivo using spectroscopic photoacoustic tomography," Proceedings of IEEE, - (2008).

[13] J.J. Niederhauser, M. Jaeger, R. Lemor, P. Weber, and M. Frenz, "Combined ultrasound and optoacoustic system for real-time high-contrast vascular imaging in vivo," IEEE Transactions on Medical Imaging, 24(4), 436-440 (2005).

[14] R.A. Kruger, W.L. Kiser, D.R. Reinecke, and G.A. Kruger, "Thermoacoustic computed tomography using a conventional linear transducer array," Medical Physics, 30(5), 856-860 (2003).

[15] K.P. Kostli, M. Frenz, H.P. Weber, G. Paltauf, and H. Schmidt-Kloiber, "Optoacoustic tomography: time-gated measurement of pressure distributions and image reconstruction," Applied Optics, 40(22), 3800-3809 (2001).

[16] E. Zhang and P. Beard, "Broadband ultrasound field mapping system using a wavelength tuned, optically scanned focused laser beam to address a Fabry Perot polymer film sensor," IEEE Transactions on Ultrasonics Ferroelectrics and Frequency Control, 53(7), 1330-1338 (2006). 
[17] R.J. Zemp, R. Bitton, M.L. Li, K.K. Shung, G. Stoica, and L.V. Wang, "Photoacoustic imaging of the microvasculature with a high-frequency ultrasound array transducer," J Biomed Opt, 12(1), 010501 (2007).

[18] J.M. Cannata, J.A. Williams, Q.F. Zhou, T.A. Ritter, and K.K. Shung, "Development of a 35-MHz piezocomposite ultrasound array for medical imaging," IEEE Transactions on Ultrasonics Ferroelectrics and Frequency Control, 53(1), 224-236 (2006). 